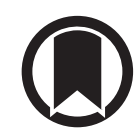

CrossMark

\title{
An inhaled "bifunctional" dual PDE3/4 inhibitor provides additional short-term improvements in lung function compared to existing classes of bronchodilator: implications for future treatment of COPD
}

\author{
Mario Cazzola ${ }^{1}$ and Clive Page ${ }^{2}$ \\ Affiliations: 'Unit of Respiratory Medicine, Dept of Experimental Medicine and Surgery, University of Rome \\ "Tor Vergata", Rome, Italy. ${ }^{2}$ Sackler Institute of Pulmonary Pharmacology, Institute of Pharmaceutical \\ Science, King's College London, London, UK. \\ Correspondence: Mario Cazzola, Unit of Respiratory Medicine, Department of Experimental Medicine and \\ Surgery, University of Rome "Tor Vergata", Via Montpellier 1, Rome 00133, Italy. E-mail mario.cazzolala
} uniroma2.it

@ERSpublications

RPL554 showed substantial bronchodilation in COPD patients but far more information from more appropriate investigations is required before we have a proper understanding of the real role that RPL554 could have in the treatment of COPD http://ow.ly/pjte30m128c

Cite this article as: Cazzola M, Page C. An inhaled "bifunctional" dual PDE3/4 inhibitor provides additional short-term improvements in lung function compared to existing classes of bronchodilator: implications for future treatment of COPD. Eur Respir J 2018; 52: 1801675 [https://doi.org/10.1183/ 13993003.01675-2018].

RPL554 is a novel inhaled dual phosphodiesterase (PDE)3/4 inhibitor that has been developed with the goal of having "bifunctional" bronchodilator and anti-inflammatory activities in a single molecule $[1,2]$. In initial exploratory clinical studies, RPL554 showed substantial bronchodilation that was comparable with peak effects reported in patients with asthma or chronic obstructive pulmonary disease (COPD) after the administration of an inhaled $\beta_{2}$-agonist, and also exhibited bronchoprotective and anti-inflammatory activities at doses having minimal adverse effects [3]

In this issue of the European Respiratory Journal, SINGH et al. [4] present the results of two phase 2 studies in which they have explored the ability of short-term treatment with RPL554 to increase the bronchodilator effects of salbutamol, ipratropium bromide or tiotropium bromide in patients with stable moderate-to-severe COPD. Overall, the two studies demonstrate that RPL554 provided additional benefits on both forced expiratory volume $1 \mathrm{~s}(\mathrm{FEV} 1)$ and measurements of hyperinflation in these patients. RPL554 was well tolerated both when administered alone or in combination with salbutamol, ipratropium or tiotropium.

The availability of an inhaled "bifunctional" dual PDE3/4 inhibitor that has both bronchodilator and anti-inflammatory activity, and that is very well tolerated, may provide a novel approach to treating patients with COPD and other inflammatory airway diseases. However, the short nature of these two phase 2 studies and the focus on measuring bronchodilation do not provide any further evidence of the real potential for this drug as a novel class of bronchodilator that also has anti-inflammatory effects. 
Whilst there is evidence of an anti-inflammatory action of RPL554 from preclinical data [1] and one study in human volunteers exposed to lipopolysaccharide published now some 5 years ago [3], to date, there are no studies demonstrating an anti-inflammatory effect in patients with COPD. However, the studies by SiNGH et al. [4] do not provide any understanding of the role this drug may play in the future armamentarium of treatments for patients with COPD. This is a missed opportunity given that the use of inhaled corticosteroids (ICS) was continued in patients in these studies if they were taking these prior to screening. Whilst short-term treatment with RPL554 is capable of providing additional improvement in lung function, these studies do not provide evidence that this drug has additional (or alternative) anti-inflammatory activity to ICS, a property that if proven, would be of great value given the current debate about the place of ICS in the treatment of most patients with COPD [5] and the increased risk of pneumonia reported for this class of drug [6].

Nonetheless, the broncholytic data with RPL554 presented by SiNGH et al. [4] confirm both preclinical $[7,8$ ] and initial clinical data [3] documenting that this drug can provide additional improvements in lung function on top of either $\beta_{2}$-agonists or muscarinic receptor antagonists, albeit in patients with some degree of reversibility. However, given that the standard of care increasingly involves the use of both a long-acting $\beta_{2}$-agonist (LABA) and a long-acting muscarinic antagonist (LAMA), often administered in combination using a single inhaler, and sometimes even triple therapy with the inclusion of an ICS, it remains to be seen what effect RPL554 may have on top of standard-of-care therapy. It is not clear from this work what is the proposed use of this new drug class. If the plan is to develop RP554 as an additional drug in the treatment of acute exacerbations of COPD, then it would also be necessary to know what RPL554 does on top of a short-acting $\beta_{2}$-agonist (SABA) and a short-acting muscarinic receptor antagonist (SAMA) at the higher doses of these drugs that are often used in this clinical setting and in more severe patients with less reversibility.

CALzeTTA et al. [7], using isolated human bronchi, documented that RPL554 is able to reduce the bronchial tone, relax airways contracted with either histamine or acetylcholine and protect the passively sensitised bronchi against the contractile action of exogenously administered histamine. In addition, RPL554 in combination with a muscarinic receptor antagonist (either atropine or glycopyrronium), and to a lesser extent with a $\beta_{2}$-adrenergic receptor agonist (salbutamol) induced a synergistic relaxation of airway smooth muscle (ASM) [7].

The ability of RPL554 to inhibit contraction of ASM induced by a variety of stimuli is likely to be due to functional antagonism analogous to that induced by $\beta_{2}$-agonists via elevation of cyclic adenosine monophosphate (cAMP) in ASM. PDE3 hydrolyses both cAMP and cyclic guanosine monophosphate (cGMP) with relatively high affinities; however, the maximal velocity for cAMP hydrolysis is nearly 10-fold higher than for cGMP [9]. PDE4 is described as a low Michaelis constant, cAMP-specific PDE with only a weak affinity for cGMP [9]. Since dual PDE3/4 inhibitors act on the same signalling pathway (i.e. they increase cAMP synthesis and block cAMP degradation, respectively), they may act together to explain the observed synergistic interactions between this drug class and $\beta_{2}$-agonists [10].

However, it is much more difficult to explain the synergistic interaction between RPL554 and atropine. Muscarinic receptor antagonists alone do not modulate the intracellular synthesis of cAMP and, in human ASM, whilst intracellular concentrations of cAMP are associated with the synergism between $\beta_{2}$-agonists and muscarinic receptor antagonists [11], adenylyl cyclase/cAMP/protein kinase A processes may not directly contribute to the functional synergistic effects observed between these two agents [12]. Furthermore, there is evidence that atropine does not only block muscarinic receptors but also directly inhibits the enzymatic activity of PDEs, in particular the cAMP-specific PDE4 [13]. This mechanism may account for increased cAMP levels, which are clearly independent of $\mathrm{M}_{1 / 2 / 3}$-muscarinic receptors, but does not involve the classical anticholinergic activity of this drug [13], although these observations could explain the synergism observed by CALzETTA et al. [7].

In the study by SINGH et al. [4], similar effects of RPL554, salbutamol and ipratropium administered alone on peak FEV1 were recorded in COPD patients, and furthermore, the peak FEV1 was higher for RPL554 plus ipratropium versus ipratropium alone and for RPL554 plus salbutamol versus salbutamol alone, but the effects were always only additive. Although this is the first study that has shown in COPD patients what has already been documented preclinically using isolated human bronchi [7], we believe that further studies are absolutely necessary to understand the pharmacological basis of the interaction between RPL554 and $\beta_{2}$-agonists, and muscarinic receptor antagonists. Furthermore, the full therapeutic potential of combined RPL554 with a SABA or a SAMA can only be established using higher doses of the SABA and a SAMA as would be used in the treatment of acute exacerbations of COPD.

Moreover, CALzETTA et al. [8] showed that RPL554 caused a clear long-lasting synergistic interaction with the muscarinic receptor antagonist glycopyrronium in enhancing the relaxant response of both medium 
and small human isolated airways, but perhaps more importantly, in extending the duration of action of the bronchodilator effect compared with administering the drugs alone. This sustained synergistic interaction occurred across the 6-h time course of the study whilst the relaxant effect of RPL554 in small airways had completely disappeared by $6 \mathrm{~h}$ post-administration and there was only a very weak effect of glycopyrronium at this time point [8].

As demonstrated in study 2 [4], RPL554 seems intended to be an add-on therapy and therefore is likely to be added on top of standard therapy. The authors have used tiotropium in this study, a once-daily bronchodilator, which is currently the most widely prescribed LAMA. Although it is likely not the intention of the owner of the drug to develop RPL554 as a (fixed) combination with tiotropium, a combination with glycopyrronium twice daily would have been a more appropriate choice to understand the importance of the clinical interaction between RPL554 and a LAMA, not only in terms of maximum bronchodilation but also of its overall amplitude (i.e. FEV1 area under the curve over $0-12 \mathrm{~h}$ ) and thus duration. We must also highlight that body plethysmography was performed pre-dose on day 1 and only pre-dose and $1.25 \mathrm{~h}$ post-dose on day 2, which did not permit the demonstration of any possible synergistic effect on the duration of bronchodilation in small airways as observed in vitro by CALzETTA et al. [8].

As stated above, it is also important to point out that the addition of RPL554 to a LABA and, above all, to a LABA/LAMA combination, which is currently the mainstay of treatment for COPD patients [14], has not yet been evaluated. Obviously, the lack of this evaluation does not negate the positive findings presented in the paper by SINGH et al. [4] but clearly far more information from more appropriate investigations is required before we have a proper understanding of the real role that RPL554 could have in the treatment of COPD.

What, however, appears inexplicable is that the clinical development of RPL554 is still at a fairly early stage, although 5 years ago, WEDZICHA [15], commenting on the first clinical results obtained with RPL554 [3], stated "Further longer term studies of RPL554 are now eagerly awaited because this could be one of the most substantial advances for some time in the management of patients with chronic airway obstruction".

Conflict of interest: M. Cazzola reports receiving grants and personal fees from Verona Pharma, and has patents 9717732, 9700558, 20160008363, 20160000790 and 20170266190 licensed to Verona Pharma. C. Page reports receiving grants and personal fees from, and holds equity in Verona Pharma.

\section{References}

1 Boswell-Smith V, Spina D, Oxford AW, et al. The pharmacology of two novel long-acting phosphodiesterase 3/4 inhibitors, RPL554 [9,10-dimethoxy-2(2,4,6-trimethylphenylimino)-3-(N-carbamoyl-2-aminoethyl)-3,4,6,7-tetrahydro2H-pyrimido[6,1-a]isoquinolin-4-one] and RPL565 [6,7-dihydro-2-(2,6-diisopropylphenoxy)-9,10-dimethoxy-4Hpyrimido[6,1-a]isoquinolin-4-one]. J Pharmacol Exp Ther 2006; 318: 840-848.

2 Abbott-Banner KH, Page CP. Dual PDE3/4 and PDE4 inhibitors: novel treatments for COPD and other inflammatory airway diseases. Basic Clin Pharmacol Toxicol 2014; 114: 365-376.

3 Franciosi LG, Diamant Z, Banner KH, et al. Efficacy and safety of RPL554, a dual PDE3 and PDE4 inhibitor, in healthy volunteers and in patients with asthma or chronic obstructive pulmonary disease: findings from four clinical trials. Lancet Respir Med 2013; 1: 714-727.

4 Singh D, Abbott-Banner K, Bengtsson T, et al. The short term bronchodilator effects of the dual PDE3 and PDE4 inhibitor RPL554 in COPD. Eur Respir J 2018.

5 Andreeva-Gateva PA, Stamenova E, Gatev T. The place of inhaled corticosteroids in the treatment of chronic obstructive pulmonary disease: a narrative review. Postgrad Med 2016; 128: 474-484.

6 Matera MG, Cardaci V, Cazzola M, et al. Safety of inhaled corticosteroids for treating chronic obstructive pulmonary disease. Expert Opin Drug Saf 2015; 14: 533-541.

7 Calzetta L, Page CP, Spina D, et al. Effect of the mixed phosphodiesterase 3/4 inhibitor RPL554 on human isolated bronchial smooth muscle tone. J Pharmacol Exp Ther 2013; 346: 414-423.

8 Calzetta L, Cazzola M, Page CP, et al. Pharmacological characterization of the interaction between the dual phosphodiesterase (PDE) 3/4 inhibitor RPL554 and glycopyrronium on human isolated bronchi and small airways. Pulm Pharmacol Ther 2015; 32: 15-23.

9 Banner KH, Press NJ. Dual PDE3/4 inhibitors as therapeutic agents for chronic obstructive pulmonary disease. Br J Pharmacol 2009; 157: 892-906.

10 Giembycz MA, Newton R. Potential mechanisms to explain how LABAs and PDE4 inhibitors enhance the clinical efficacy of glucocorticoids in inflammatory lung diseases. F1000Prime Rep 2015; 7: 16.

11 Calzetta L, Matera MG, Cazzola M. Pharmacological mechanisms leading to synergy in fixed-dose dual bronchodilator therapy. Curr Opin Pharmacol 2018; 40: 95-103.

12 Kume $\mathrm{H}$, Nishiyama $\mathrm{O}$, Isoya $\mathrm{T}$, et al. Involvement of allosteric effect and $\mathrm{K}_{\mathrm{Ca}}$ channels in crosstalk between $\beta_{2}$-adrenergic and muscarinic $\mathrm{M}_{2}$ receptors in airway smooth muscle. Int J Mol Sci 2018; 19: E1999.

13 Perera RK, Fischer TH, Wagner M, et al. Atropine augments cardiac contractility by inhibiting cAMP-specific phosphodiesterase type 4. Sci Rep 2017; 7: 15222.

14 Vogelmeier CF, Criner GJ, Martinez FJ, et al. Global Strategy for the Diagnosis, Management, and Prevention of Chronic Obstructive Lung Disease 2017 Report: GOLD Executive Summary. Eur Respir J 2017; 49: 1700214.

15 Wedzicha JA. Dual PDE 3/4 inhibition: a novel approach to airway disease? Lancet Respir Med 2013; 1: 669-670 